\title{
Incidência de fungos e quantificação de danos em sementes de genótipos de arroz
}

\author{
Vanda Maria Angeli Malavolta ${ }^{1}$, Elaine de Arruda Soligo², Débora Daiana Dias ${ }^{3}$, \\ Luiz Ernesto Azzini ${ }^{1}$, Cândido Ricardo Bastos ${ }^{1}$
}

${ }^{1}$ Instituto Agronômico/ IAC, Centro de Grãos e Fibras. CP28, Campinas, SP. E-mail: vanda@iac.sp.gov.br; ${ }^{2}$ Estagiária, Bolsista do CNPq/PIBIC em 2003/04; ${ }^{3}$ Estagiária, Bolsista do CNPq/PIBIC em 2004/05.

Trabalho parcialmente financiado pela FAPESP.

Autor(a) para correspondência: Vanda M.A.Malavolta.

Data de chegada: 27/10/2005. Aceito para publicação em: 11/10/2006.

\section{RESUMO}

Malavolta, V.M.A.; Soligo, E.A.; Dias, D.D.; Azzini, L.E.; Bastos, C.R. Incidência de fungos e quantificação de danos em sementes de genótipos de arroz. Summa Phytopathologica, v.33, n.3, p.280-286, 2007.

O programa de melhoramento genético de arroz do Instituto Agronômico visa a obtenção de cultivares de arroz altamente produtivas, características tecnológicas desejáveis e resistência a doenças. O presente estudo avaliou genótipos de arroz integrantes dos ensaios comparativos avançados quanto à qualidade sanitária e severidade de manchas nas sementes, peso das sementes por panícula e esterilidade das espiguetas. A qualidade sanitária das sementes variou conforme o sistema de cultivo, com alta incidência dos fungos Pyricularia grisea, Bipolaris oryzae e Microdochium oryzae no cultivo inundado, enquanto que sob irrigação por aspersão predominaram Pyricularia grisea, Phoma sorghina e Drechslera spp. A severidade de manchas nas sementes apresentou correlação negativa com peso da panícula e número de grãos cheios, e positiva com número de grãos chochos e porcentagem de esterilidade. Destacaram-se em condições de cultivo irrigado por inundação as linhagens IAC 1817 e IAC 1818, apresentando os menores índices de severidade de manchas, e também baixas porcentagens de esterilidade e altos pesos de grãos por panícula. Em terras altas sob irrigação por aspersão, destacaram-se a linhagem IAC 1776 e a cv. Bonança, com os menores índices de severidade de manchas, e a linhagem 1781, com baixas incidências dos mais importantes patógenos, e também baixa esterilidade. Esses resultados serviram como indicadores do nível de resistência e/ou tolerância dos genótipos a manchas de grãos.

Palavras-chave adicionais: Oryza sativa, mancha de grãos, resistência genética, controle, sanidade de sementes.

\begin{abstract}
Malavolta, V.M.A.; Soligo, E.A.; Dias, D.D.; Azzini, L.E.; Bastos, C.R. Fungi incidence and damage evaluation on seeds of rice genotypes . Summa Phytopathologica, v.33, n.3, p.280-286, 2007.

Aiming the development of high yielding rice cultivars, IAC (Agronomic Institute of S. Paulo, Brazil) carries out a continuous breeding program focused on good-grain quality and disease resistance. Rice genotypes from the upland and irrigated advanced trials were evaluated for seed health, seed discoloration severity, panicle seed weight and spikelet sterility. The results showed that seed health varied according to the two environments. High seed incidence of Pyricularia grisea, Bipolaris oryzae and Microdochium oryzae occurred under irrigated conditions, whereas Pyricularia grisea, Phoma

sorghina e Drechslera spp. in upland. Correlation coefficient values obtained were significant and negative for seed discoloration severity $\mathrm{x}$ panicle seed weight and filled grains, and positive for seed discoloration severity $\mathrm{x}$ empty spikelets and spikelet sterility. In lowlands, the lines IAC 1817 e IAC 1818 showed low values of seed discoloration severity, spikelet sterility and high seed weight per panicle. In upland, IAC 1776 line and cv. Bonança showed the lowest seed discoloration severity, while IAC 1781 line presented low incidences of the most important pathogens, and low spikelet sterility.
\end{abstract}

Additional Keywords: Oryza sativa, grain discoloration, genetic resistance, control, seed health.

O arroz é alimento fundamental da população brasileira, constituindo-se na base da dieta alimentar e conseqüentemente de grande importância sócio-econômica para a economia nacional.

No Estado de São Paulo a produção de arroz tem oscilado, nestes últimos anos, ao redor de 100 mil toneladas de arroz em casca, representando pequena parte das necessidades de consumo. O Brasil tem apresentado produções anuais na faixa de 11 a 13 milhões de toneladas, e seu consumo está muito próximo a 13 milhões de toneladas
(11). Diante dessa realidade, torna-se necessário a adoção de medidas que visem o aumento da produção de arroz, principalmente no Estado de São Paulo. A utilização de cultivares melhoradas é considerada um dos meios mais eficazes de se aumentar a produtividade, constituindose na tecnologia de menor custo para o agricultor, de fácil adoção e que não agride o meio ambiente.

O programa de melhoramento genético do arroz do Instituto Agronômico, iniciado em 1935, tem alcançado inúmeros sucessos 
através dos anos. Atualmente, esse programa visa a obtenção de novas cultivares de arroz altamente produtivas, com bom rendimento no beneficiamento, aliadas a um bom tipo de grão (longo, fino e cristalino) e resistência à doenças.

Entre as doenças que incidem no arroz, as manchas de grãos constituem um problema comum, e que vem ocorrendo há muito tempo em diversos países produtores (13). Mais recentemente, sua crescente importância vem sendo relatada na Indonésia, Índia e Estados Unidos, principalmente em cultivos de terras altas, onde o problema vem assumindo sérias proporções $(4,7,21)$.

No Brasil, a ocorrência de manchas de grãos também vem aumentando nos últimos anos, tendo assumido posição de doença economicamente importante devido à maior suscetibilidade da maioria das cultivares atualmente em uso. Nos últimos anos, entre as doenças mais importantes do arroz na região sul do Brasil, as manchas de glumas estão em segundo lugar, logo depois da brusone (6). No Estado de Tocantins, as manchas de grãos foram consideradas em 1996 como o problema fitossanitário de maior importância (17). A importância das manchas de grãos e a maior suscetibilidade das cultivares empregadas nos últimos anos foi relatada no Rio Grande do Sul (15), na microrregião do Alto Purus, Acre (3), em Goiás (28), nos Estados de Goiás, Mato Grosso e Mato Grosso do Sul (1, 24, 26), em Mato Grosso e Rondônia (23), nos Estados de Goiás e Tocantins $(5,18,19)$, em Minas Gerais (16). Em São Paulo, no Vale do Paraíba, principal região produtora de arroz irrigado, a ocorrência de fungos causadores de manchas de grãos vem causando há muitos anos descoloração e redução do peso em sementes de arroz (22). Levantamento realizado mais recentemente confirmou a alta incidência desses fungos nos campos de produção de sementes básicas e certificadas de arroz daquela região (8).

A etiologia da doença mancha de grãos é complexa, e envolve geralmente vários fungos ocorrendo simultaneamente. Os mais comuns e constatados com maior freqüência são Bipolaris oryzae (=Helminthosporium oryzae), Pyricularia grisea, Phoma sorghina, Microdochium oryzae (=Rhynchosporium oryzae), Alternaria padwickii, Alternaria spp., Curvularia lunata e Nigrospora oryzae.

A extensão dos sintomas ocasionados pela incidência dos fungos manchadores de grãos varia grandemente de acordo com o patógeno predominante, condições climáticas e localidade (13), assim como seus efeitos nos componentes da produção $(21,22,30)$.

Entre as medidas de controle recomendadas para manchas de grãos de arroz estão a adoção de práticas culturais preventivas, tais como utilização de cultivares mais resistentes ou tolerantes e adubação equilibrada, e complementarmente, a aplicação de fungicidas $(6,15)$. A utilização de cultivares com maior nível de resistência / tolerância é, sem dúvida, a medida mais importante, motivo pelo qual a avaliação e seleção de materiais resistentes vem sendo continuamente realizada em novos genótipos $(9,17,25,27)$.

Uma das formas de selecionar genótipos resistentes à manchas de grãos é a avaliação das sementes colhidas quanto à incidência e severidade de manchas nas mesmas e identificação dos patógenos responsáveis pelos sintomas $(2,10,20,23)$. Como o trabalho de melhoramento do arroz é um processo que prevê a obtenção contínua de novos genótipos de arroz com melhores características agronômicas e resistência a doenças, essas avaliações também devem ser realizadas de forma contínua.

O objetivo do presente trabalho foi a avaliação de novas linhagens de arroz, integrantes dos Ensaios Avançados do Programa de Melhoramento do Arroz do IAC, quanto ao nível de resistência e/ou tolerância à manchas de grãos.

\section{MATERIAL E MÉTODOS}

Foram instalados nos municípios de Taubaté (distrito de Quiririm) e Capão Bonito, ambos no Estado de São Paulo, nos anos agrícolas 2002/03 e 2003/2004, ensaios comparativos avançados do Programa de Melhoramento de Arroz do Instituto Agronômico que incluem linhagens previamente selecionadas e cultivares comerciais para servirem de parâmetros comparativos. Esses ensaios destinam-se à avaliação final de linhagens elite e definição dos genótipos que deverão ser lançados como novas cultivares, servindo também como fonte de dados para a determinação do Valor de Cultivo e Uso (VCU), necessário para o registro das novas cultivares.

No primeiro ano avaliou-se 14 genótipos procedentes do município de Taubaté, em condições de cultivo irrigado por inundação: EPAGRI 109, IAC 101, IAC 103, IAC 1788, IAC 1791, IAC 1795 , IAC 1798, IAC 1810, IAC 1811, IAC 1813, IAC 1817, IAC 1818, SCS-BRS 111, SCS 112. No segundo ano, além desses, foram avaliados outros 8 genótipos: IAC 400, IAC 1844, IAC 1847, IAC 1851 , IAC 1852, IAC 1854, IAC 1857 e SCS-BRS 113. Procedentes do município de Capão Bonito, em cultivo de terras altas sob irrigação por aspersão, avaliou-se 16 genótipos nos 2 anos agrícolas: Bonança, Carisma, CNA 8983, IAC 201, IAC 202, IAC 1652, IAC 1711, IAC 1732, IAC 1738, IAC 1740, IAC 1744, IAC 1774, IAC 1776, IAC 1779, IAC 1781 e Primavera.

O delineamento experimental utilizado foi blocos casualizados, com 4 repetições. As parcelas experimentais foram constituídas por 7 linhas de $5 \mathrm{~m}$ de comprimento. A adubação basal foi de $400 \mathrm{~kg} \cdot \mathrm{ha}^{-1} \mathrm{da}$ mistura 4-14-8.

Nas condições de cultivo irrigado por inundação, foi realizado o plantio de mudas em 14/01/03 e 07/01/04 no primeiro e segundo ano, respectivamente, no espaçamento de $0,20 \mathrm{~m}$ entre linhas e $0,20 \mathrm{~m}$ entre covas na linha. A semeadura para o preparo dessas mudas ocorreu em 31/12/02 e 26/12/03. Nos 2 anos efetuou-se 1 aplicação nitrogenada em cobertura, aproximadamente aos 30 dias pós transplantio, empregando-se $40 \mathrm{~kg}$ de nitrogênio.ha ${ }^{-1}$. No cultivo de terras altas, a semeadura foi realizada em 20/11/02 e 21/11/03, utilizando-se espaçamento de $0,50 \mathrm{~m}$ entre linhas e densidade de semeadura de 160 sementes.m-2. Foram feitas 2 adubações nitrogenadas em cobertura, aos 40 e 60 dias pós-emergência, empregando-se $20 \mathrm{~kg}$ de nitrogênio. ha-1.

\section{Foram realizadas as seguintes avaliações:}

1) Peso dos grãos produzidos por panícula (média de 10 panículas).

2) Número de grãos cheios e grãos chochos por panícula (média de 10 panículas), e cálculo das respectivas porcentagens de esterilidade.

3) Severidade de manchas, determinada em amostra constituída por 100 sementes, utilizando-se a escala anteriormente utilizada (14) e ligeiramente modificada, onde: $\mathbf{0}=$ sem sintoma, $\mathbf{1}=$ glumas com manchas pequenas, tipo "cabeça de alfinete", $\mathbf{2}=$ manchas atingindo até $25 \%$ da superfície do grão, $3=$ manchas atingindo de 26 a $50 \%$ da superfície do grão, $4=$ manchas atingindo mais de $50 \%$ da superfície do grão. A partir dos dados de severidade de manchas nos grãos, foi calculado o índice de severidade da doença pela seguinte fórmula:

ISD $=\sum$ (Nota $x$ freqüência $) / n .^{\circ}$ total de grãos

4) Sanidade de sementes, através do teste de papel de filtro com congelamento (12), em amostras de 200 sementes para cada repetição.

Os resultados das avaliações foram submetidos a análises de variância utilizando o sistema de análise estatística SANEST (29). A comparação das médias foi feita pelo Teste de Tukey, a 5\% de probabilidade. 


\section{RESULTADOS E DISCUSSÃO}

Avaliações das sementes produzidas em Taubaté, condições de cultivo irrigado por inundação:

A maioria das variáveis analisadas apresentou significância pelo Teste F para a interação ano x genótipo, motivo pelo qual foram considerados as análises e comparação de médias dentro de cada ano. Para as variáveis constituídas pela incidência dos fungos Bipolaris oryzae, Alternaria alternata, Fusarium sp. e Nigrospora oryzae, que não apresentaram interação significativa ano x genótipo, foram considerados os resultados médios para os genótipos comuns aos dois anos para a comparação de médias. Independente do resultado da análise conjunta, sempre foi apresentada a comparação de médias no segundo ano, devido à inclusão nesse ano de oito novos genótipos nos ensaios.

Os testes de sanidade de sementes (Tabela 1) mostram no primeiro ano elevada incidência de Pyricularia grisea, Bipolaris oryzae, Microdochium oryzae, Phoma sorghina e Drechslera spp., importantes patógenos do arroz, com incidências médias de 20,0; 13,$6 ; 24,6 ; 28,6$ e $31,7 \%$ respectivamente. O fungo Alternaria padwickii, considerado um patógeno de menor agressividade que os anteriores, ocorreu em intensidade mais baixa, apresentando incidência média de $8,2 \%$. No segundo ano observou-se novamente elevada incidência de Pyricularia grisea, Microdochium oryzae, Phoma sorghina e Drechslera spp. ,com incidências médias de 14,2; 16,9; 29,8 e $23,4 \%$ respectivamente. Por outro lado, os fungos Bipolaris oryzae e Alternaria padwickii ocorreram em intensidades bem mais baixas, com incidência média de $1,6 \%$ e $0,3 \%$ respectivamente.

Os demais fungos constatados - Alternaria alternata, Cladosporium sp., Epicoccum sp., Curvularia spp., Fusarium sp. e Nigrospora oryzae - são de ocorrência muito comum em sementes de arroz, embora sejam de baixa patogenicidade e raramente responsáveis por má germinação das sementes ou baixo vigor de plântulas, embora sejam comumente relacionados como integrantes do complexo causador de descoloração de sementes de arroz.

Observa-se pelas médias anuais que, de maneira geral, a incidência de fungos no segundo ano foi menor que no primeiro. Esse decréscimo foi muito acentuado principalmente para $B$. oryzae e $A$. padwickii, que tiveram a porcentagem de ocorrência reduzida a menos de $10 \%$ em relação ao ano anterior.

Para a discussão dos dados, optou-se por destacar os genótipos com maiores ou menores valores absolutos, dependendo da variável analisada, mesmo quando, em alguns casos, eles não tenham diferido estatisticamente de alguns genótipos com comportamento intermediário.

As sementes dos genótipos apresentaram comportamento variável como hospedeiras dos fungos constatados. Observa-se que as menores incidências de $P$. grisea ocorreram em IAC 1791, IAC 1798, IAC 1810, IAC 1817, IAC 1818 e SCS-BRS 111 nos 2 anos de observação, e também por IAC 1844, IAC 1847, IAC 1851, IAC 1854 e IAC 1857 no segundo ano. As menores incidências médias com relação à $B$. oryzae, foram registradas em IAC 103 e SCS 111. Com relação à $P$. sorghina destacaram-se no primeiro ano IAC 1795 e IAC 1818, e no segundo ano IAC 101, IAC 103, IAC 1788, IAC 1851, IAC 1852 e IAC 1857. Com relação à $M$. oryzae, IAC 1788 nos 2 anos e também IAC 1818 no segundo ano. Com relação a Drechslera spp, IAC 1798 nos 2 anos. Com relação à $A$. padwickii, IAC 1788 e IAC 1791 no primeiro ano.

Observa-se na Tabela 2 que a severidade de manchas, traduzida pelo índice de severidade de doença (ISD) apresentou amplitude de variação entre 1,28 e 2,01 (escala de 0-4) no primeiro ano, e entre 1,40 e 2,65 no segundo ano, podendo-se diferenciar

Tabela 1. Incidência dos principais fungos (\%) constatados em sementes de arroz, detectadas através do teste de papel de filtro com congelamento. Taubaté, anos agrícolas 2002/2003 e 2003/2004.

\begin{tabular}{|c|c|c|c|c|c|c|c|c|c|c|c|c|c|c|c|c|c|c|}
\hline \multirow{3}{*}{ Genótipos } & \multicolumn{18}{|c|}{ FUNGOS } \\
\hline & \multicolumn{3}{|c|}{ Pyricularia grisea } & \multicolumn{3}{|c|}{ Bipolaris oryzae } & \multicolumn{3}{|c|}{ Microdochium oryzae } & \multicolumn{3}{|c|}{ Phoma sorghina } & \multicolumn{3}{|c|}{ Drechslera spp. } & \multicolumn{3}{|c|}{ Alternaria padwickii } \\
\hline & Ano 1 & Ano 2 & Média & Ano 1 & Ano 2 & Média & Ano 1 & Ano 2 & Média & Ano 1 & Ano 2 & Média & Ano 1 & Ano 2 & Média & Ano 1 & Ano 2 & Média \\
\hline Epagri 109 & 29,6 ab & $31,3 \mathrm{ab}$ & 30,4 & 15,0 & $1,3 \mathrm{ab}$ & 8,1 ab & $16,9 \mathrm{c}-\mathrm{e}$ & $15,3 \mathrm{bd}$ & 16,1 & $26,4 \mathrm{ab}$ & $28,6 \mathrm{ab}$ & 27,5 & $25,9 \mathrm{c}-\mathrm{f}$ & $15,8 \mathrm{c}$ & 20,8 & $4,1 \mathrm{c}-\mathrm{f}$ & 0,3 a & 2,2 \\
\hline IAC 101 & 34,1 a & $12,9 \mathrm{~d}-\mathrm{g}$ & 23,5 & 15,0 & $1,0 \mathrm{ab}$ & $7,8 a b$ & $16,4 \mathrm{c}-\mathrm{e}$ & 15,5 b-d & 16,0 & $25,4 a b$ & $22,1 \mathrm{~b}$ & 23,8 & 33,3 a-e & $21,8 \mathrm{a}-\mathrm{c}$ & 27,5 & 6,4 b-f & $1,0 \mathrm{a}$ & 3,7 \\
\hline IAC 103 & 36,8 a & $11,3 \mathrm{~d}-\mathrm{h}$ & 24,0 & 6,3 & $0,5 \mathrm{ab}$ & 3,4 b & 23,5 b-e & 14,0 b-d & 18,7 & $30,4 \mathrm{ab}$ & $23,8 \mathrm{~b}$ & 27,1 & $25,0 \mathrm{~d}-\mathrm{f}$ & $21,5 \mathrm{a}-\mathrm{c}$ & 23,2 & 9,8 a-e & $0,3 \mathrm{a}$ & 5,0 \\
\hline IAC 1788 & 26,6 a-c & $47,6 \mathrm{a}$ & 37,1 & 12,5 & $1,3 \mathrm{ab}$ & $6,9 \mathrm{ab}$ & $11,4 \mathrm{e}$ & $8,4 \mathrm{~d}$ & 9,9 & $25,3 \mathrm{ab}$ & $23,5 \mathrm{~b}$ & 24,4 & $19,0 \mathrm{f}$ & $27,8 \mathrm{a}-\mathrm{c}$ & 23,4 & $2,6 \mathrm{f}$ & $0,5 \mathrm{a}$ & 1,6 \\
\hline IAC 1791 & 16,4 b-e & 9,3 e-i & 12,8 & 12,9 & $1,9 \mathrm{ab}$ & $7,4 a b$ & 20,5 b-e & 19,9 a-c & 20,2 & $26,8 \mathrm{ab}$ & $33,1 \mathrm{ab}$ & 30,0 & $24,8 \mathrm{~d}-\mathrm{f}$ & 34,1 a & 29,5 & $2,8 \mathrm{ef}$ & $0,0 \mathrm{a}$ & 1,4 \\
\hline IAC 1795 & 21 a-d & 41,6 a & 31,3 & 14,3 & $0,5 \mathrm{ab}$ & $7,4 a b$ & $13,6 \mathrm{de}$ & $20,1 \mathrm{a}-\mathrm{c}$ & 16,9 & 20,2 b & $27,4 a b$ & 23,8 & 28,3 b-f & $30,1 \mathrm{a}-\mathrm{c}$ & 29,2 & $4,0 \mathrm{~d}-\mathrm{f}$ & 0,1 a & 2,1 \\
\hline IAC 1798 & $7,4 \mathrm{e}$ & $2,9 \mathrm{i}$ & 5,1 & 9,3 & $0,5 \mathrm{ab}$ & $4,9 a b$ & $30,8 \mathrm{bc}$ & 20,9 a-c & 25,8 & $30,1 \mathrm{ab}$ & $35,4 \mathrm{ab}$ & 32,8 & 21,0 ef & $15,8 \mathrm{c}$ & 18,4 & $5,3 \mathrm{c}-\mathrm{f}$ & $0,0 \mathrm{a}$ & 2,7 \\
\hline IAC 1810 & $11,9 \mathrm{c}-\mathrm{e}$ & $6,3 \mathrm{f}-\mathrm{i}$ & 9,1 & 23,9 & $0,6 \mathrm{ab}$ & $12,3 \mathrm{a}$ & $29,3 \mathrm{bc}$ & $12,6 \mathrm{~cd}$ & 21,0 & $33,3 \mathrm{ab}$ & $32,0 \mathrm{ab}$ & 32,7 & $43,0 \mathrm{ab}$ & $25,4 \mathrm{a}-\mathrm{c}$ & 34,2 & 12,0 a-d & 0,1 a & 6,1 \\
\hline IAC 1811 & 35,9 a & 23,8 b-d & 29,8 & 15,9 & $4,8 \mathrm{ab}$ & $10,3 \mathrm{a}$ & 20,1 b-e & 13,9 b-d & 17,0 & $28,0 \mathrm{ab}$ & $26,1 \mathrm{ab}$ & 27,1 & 39,1 a-d & 24,6 a-c & 31,9 & 6,4 b-f & $0,6 \mathrm{a}$ & 3,5 \\
\hline IAC 1813 & $5,1 \mathrm{e}$ & $16,3 \mathrm{c}-\mathrm{f}$ & 10,7 & 16,5 & $0,8 \mathrm{ab}$ & $8,1 \mathrm{ab}$ & 52,1 a & $11,1 \mathrm{~cd}$ & 31,6 & 44,1 a & $31,0 \mathrm{ab}$ & 37,6 & 48,9 a & 24,6 a-c & 36,8 & 16,8 a & $0,0 \mathrm{a}$ & 8,4 \\
\hline IAC 1817 & $7,3 \mathrm{e}$ & $3,8 \mathrm{hi}$ & 5,5 & 18,9 & $2,8 \mathrm{ab}$ & 10,8 a & $32,9 \mathrm{ab}$ & $21,0 \mathrm{a}-\mathrm{c}$ & 27,0 & $28,1 \mathrm{ab}$ & $27,5 \mathrm{ab}$ & 27,8 & $41,5 \mathrm{a}-\mathrm{c}$ & 23,4 a-c & 32,4 & $14,4 \mathrm{ab}$ & $1,5 \mathrm{a}$ & 8,0 \\
\hline IAC 1818 & $7,9 \mathrm{de}$ & $2,4 \mathrm{i}$ & 5,1 & 14,6 & $1,5 \mathrm{ab}$ & $8,1 \mathrm{ab}$ & 26,4 b-d & $8,9 \mathrm{~d}$ & 17,6 & $22,8 \quad$ b & $44,5 \mathrm{a}$ & 33,7 & 37,5 a-d & $18,6 \mathrm{a}-\mathrm{c}$ & 28,1 & $12,5 \mathrm{a}-\mathrm{c}$ & 0,6 a & 6,6 \\
\hline SCS 112 & $33,9 \mathrm{ab}$ & 22,3 b-d & 28,1 & 7,5 & $1,0 \mathrm{ab}$ & $4,3 \mathrm{ab}$ & 18,1 b-e & 13,8 b-d & 15,9 & $30,3 \mathrm{ab}$ & $32,8 \mathrm{ab}$ & 31,5 & 25,4 d-f & $16,6 \mathrm{bc}$ & 21,0 & 4,3 c-f & 0,1 a & 2,2 \\
\hline SCS-Brs111 & $6,5 \mathrm{e}$ & $3 \mathrm{i}$ & 4,8 & 8,3 & $0,0 \mathrm{~b}$ & $4,2 \mathrm{~b}$ & $32,3 \mathrm{~b}$ & $11,5 \mathrm{~cd}$ & 21,9 & $29,6 \mathrm{ab}$ & $37,4 a b$ & 33,5 & 31,0 b-f & $19,3 \mathrm{a}-\mathrm{c}$ & 25,1 & $13,8 \mathrm{ab}$ & 0,1 a & 7,0 \\
\hline IAC 400 & & $10,8 \mathrm{~d}-\mathrm{h}$ & & & $1,0 \mathrm{ab}$ & & & 13,8 b-d & & & $36,5 \mathrm{ab}$ & & & $22,3 \mathrm{a}-\mathrm{c}$ & & & $0,1 \mathrm{a}$ & \\
\hline IAC 1844 & & $3,8 \mathrm{hi}$ & & & $0,6 \mathrm{ab}$ & & & 18,0 a-d & & & $31,0 \mathrm{ab}$ & & & 19,4 a-c & & & $0,6 \mathrm{a}$ & \\
\hline IAC 1847 & & $2,1 \mathrm{i}$ & & & $1,5 \mathrm{ab}$ & & & 18,3 a-d & & & $34,4 \mathrm{ab}$ & & & 26,4 a-c & & & $0,3 \mathrm{a}$ & \\
\hline IAC 1851 & & $5,4 \mathrm{~g}-\mathrm{i}$ & & & $4,6 \mathrm{a}$ & & & 29,1 a & & & $21,1 \mathrm{~b}$ & & & $26,8 \mathrm{a}-\mathrm{c}$ & & & $0,0 \mathrm{a}$ & \\
\hline IAC 1852 & & $17,1 \mathrm{~b}-\mathrm{e}$ & & & $4,5 \mathrm{ab}$ & & & 29,0 a & & & $23,3 \mathrm{~b}$ & & & 23,3 a-c & & & $0,3 \mathrm{a}$ & \\
\hline IAC 1854 & & $6,3 \mathrm{e}-\mathrm{i}$ & & & $3,1 \mathrm{ab}$ & & & $25,3 \mathrm{ab}$ & & & $26,4 \mathrm{ab}$ & & & $31,8 \mathrm{ab}$ & & & $0,5 \mathrm{a}$ & \\
\hline IAC 1857 & & $2,2 \mathrm{i}$ & & & $1,5 \mathrm{ab}$ & & & 19,7 a-c & & & 19,3 b & & & 24,5 a-c & & & $0,0 \mathrm{a}$ & \\
\hline SCSBRS 113 & & 29,8 a-c & & & $1,1 \mathrm{ab}$ & & & $11,8 \mathrm{~cd}$ & & & $38,3 \mathrm{ab}$ & & & $21,9 \mathrm{a}-\mathrm{c}$ & & & $0,3 \mathrm{a}$ & \\
\hline Média & 20,0 & 14,2 & 18,4 & 13,6 & 1,6 & 7,4 & 24,6 & 16,9 & 19,7 & 28,6 & 29,8 & 29,5 & 31,7 & 23,4 & 27,2 & 8,2 & 0,3 & 4,3 \\
\hline CV $(\%)$ & 17,0 & 16,9 & & & 38,1 & 25,7 & 12,3 & 12,6 & & 10,8 & 12,0 & & 9,5 & 11,9 & & 21,0 & 32,1 & \\
\hline
\end{tabular}

Médias seguidas da mesma letra, dentro de cada coluna, não diferem estatisticamente entre si pelo teste de Tukey a $5 \%$. 
Tabela 2. Índice de Severidade de manchas(ISD) e características agronômicas apresentadas por genótipos de arroz. Taubaté, anos agrícolas 2002/2003 e $2003 / 2004$.

\begin{tabular}{|c|c|c|c|c|c|c|c|c|c|}
\hline \multirow[t]{2}{*}{ Genótipos } & \multicolumn{3}{|c|}{ ISD } & \multicolumn{3}{|c|}{ Peso por panícula } & \multicolumn{3}{|c|}{ Esterilidade(\%) } \\
\hline & Ano 1 & Ano 2 & Média & Ano 1 & Ano 2 & Média & Ano 1 & Ano 2 & Média \\
\hline Epagri 109 & $1,66 \mathrm{ab}$ & 1,78 b-e & 1,72 & $1,70 \mathrm{~cd}$ & $1,42 \mathrm{~d}-\mathrm{j}$ & 1,56 & 30,0 b-e & $35,4 \mathrm{f}-\mathrm{j}$ & 32,7 \\
\hline IAC 101 & $1,46 a b$ & 2,10 a-e & 1,78 & 2,13 a-d & $1,54 \mathrm{c}-\mathrm{h}$ & 1,83 & $25,5 \mathrm{c}-\mathrm{e}$ & $31,3 \mathrm{~h}-\mathrm{j}$ & 28,4 \\
\hline IAC 103 & $1,65 a b$ & 2,25 a-c & 1,95 & $1,65 \mathrm{~cd}$ & $0,58 \mathrm{k}$ & 1,11 & $25,8 \mathrm{c}-\mathrm{e}$ & 58,6 ab & 42,2 \\
\hline IAC 1788 & $1,90 \mathrm{ab}$ & 2,12 a-e & 2,01 & 1,85 b-d & $1,42 \mathrm{~d}-\mathrm{j}$ & 1,63 & 46,6 a & $46,1 \mathrm{c}-\mathrm{e}$ & 46,4 \\
\hline IAC 1791 & $1,76 \mathrm{ab}$ & 1,90 b-e & 1,83 & $1,53 \mathrm{~d}$ & 1,64 b-f & 1,58 & $43,9 \mathrm{ab}$ & 40,6 d-h & 42,2 \\
\hline IAC 1795 & $1,73 \mathrm{ab}$ & 2,10 a-e & 1,91 & 2,10 a-d & $1,81 \mathrm{bc}$ & 1,95 & 32,9 a-c & $39,7 \mathrm{~d}-\mathrm{i}$ & 36,3 \\
\hline IAC 1798 & $1,69 \mathrm{ab}$ & $2,46 \mathrm{ab}$ & 2,07 & 2,23 a-c & $1,23 \mathrm{gj}$ & 1,73 & 30,6 b-d & $53,9 \mathrm{bc}$ & 42,3 \\
\hline IAC 1810 & $1,62 \mathrm{ab}$ & $2,65 \mathrm{a}$ & 2,13 & $2,45 \mathrm{ab}$ & $1,46 \mathrm{cj}$ & 1,95 & $19,6 \mathrm{c}-\mathrm{e}$ & $33,4 \mathrm{~g}-\mathrm{j}$ & 26,5 \\
\hline IAC 1811 & $1,55 \mathrm{ab}$ & 1,95 a-e & 1,75 & $2,00 \mathrm{bd}$ & $1,41 \mathrm{~d}-\mathrm{j}$ & 1,70 & 26,6 c-e & $39,3 \mathrm{~d}-\mathrm{i}$ & 32,9 \\
\hline IAC 1813 & $2,01 \mathrm{a}$ & 1,84 b-e & 1,92 & 2,03 b-d & $1,40 \mathrm{e}-\mathrm{j}$ & 1,71 & 20,9 c-e & $31,3 \mathrm{hj}$ & 26,1 \\
\hline IAC 1817 & $1,44 a b$ & $1,40 \mathrm{e}$ & 1,42 & $2,70 \mathrm{a}$ & 1,59 b-g & 2,14 & $18,2 \mathrm{de}$ & $39,0 \mathrm{e}-\mathrm{i}$ & 28,6 \\
\hline IAC 1818 & $1,28 \quad b$ & $1,56 \mathrm{c}-\mathrm{e}$ & 1,42 & $2,38 a b$ & $1,17 \mathrm{ij}$ & 1,77 & $16,4 \mathrm{e}$ & 43,8 d-f & 30,1 \\
\hline SCS 112 & $1,61 \mathrm{ab}$ & 1,90 b-e & 1,75 & $1,73 \mathrm{~cd}$ & $1,13 \mathrm{j}$ & 1,43 & 28,4 c-e & $29,1 \mathrm{j}$ & 28,7 \\
\hline SCS-Brs111 & $1,44 a b$ & 2,24 a-c & 1,84 & $1,68 \mathrm{~cd}$ & $0,54 \mathrm{k}$ & 1,11 & 26,9 c-e & 66,8 a & 46,8 \\
\hline IAC 400 & & 2,08 a-e & & & $1,53 \mathrm{c}-\mathrm{i}$ & & & $37,8 \mathrm{e}-\mathrm{j}$ & \\
\hline IAC 1844 & & $1,49 \mathrm{de}$ & & & $1,37 \mathrm{e}-\mathrm{j}$ & & & $30,4 \mathrm{ij}$ & \\
\hline IAC 1847 & & 1,97 a-e & & & $1,32 \mathrm{f}-\mathrm{j}$ & & & $45,5 \mathrm{c}-\mathrm{e}$ & \\
\hline IAC 1851 & & 1,75 b-e & & & 1,73 b-e & & & $48,7 \mathrm{~cd}$ & \\
\hline IAC 1852 & & 1,92 b-e & & & $1,93 \mathrm{~b}$ & & & $45,8 \mathrm{c}-\mathrm{e}$ & \\
\hline IAC 1854 & & 2,15 a-d & & & $2,42 \mathrm{a}$ & & & 42,2 d-g & \\
\hline IAC 1857 & & 2,08 a-e & & & $1,77 \mathrm{~b}-\mathrm{d}$ & & & 46,1 c-e & \\
\hline SCSBRS 113 & & 2,04 a-e & & & $1,20 \mathrm{~h}-\mathrm{j}$ & & & $35,1 \mathrm{f}-\mathrm{j}$ & \\
\hline Média & 1,6 & 2,0 & 1,82 & 2,0 & 1,4 & 1,65 & 28,0 & 41,8 & 35,0 \\
\hline CV (\%) & 16,8 & 13,7 & & 12,7 & 9,5 & & 19,8 & 8,6 & \\
\hline
\end{tabular}

Médias seguidas da mesma letra, dentro de cada coluna, não diferem estatisticamente entre si pelo teste de Tukey a 5\%.

estatisticamente esses valores. O menor ISD foi apresentado por IAC 1818 no primeiro ano, e também no segundo ano, juntamente com IAC 1817 e IAC 1844.

Ressalta-se que os genótipos IAC 1817 e IAC 1818 apresentaram os menores ISD, e também baixas porcentagens de esterilidade e os maiores pesos por panícula, constituindo materiais promissores quanto à resistência/tolerância à manchas de grãos.

Pelas médias anuais pode-se observar que, em relação ao primeiro ano, ocorreu no segundo ano um aumento nos valores de severidade de manchas nas sementes (ISD) e esterilidade, e decréscimo no peso das sementes, evidenciando efeito nocivo da incidência de manchas. Por outro lado, também ocorreu conforme comentado anteriormente, decréscimo no segundo ano em relação ao primeiro na incidência média de fungos nas sementes, evidenciando que a severidade de manchas está melhor relacionada às características agronômicas que a simples incidência de patógenos nas sementes.

Essa redução na incidência de fungos deve-se provavelmente à prevalência de condições climáticas menos favoráveis à disseminação dos patógenos, embora favoráveis ao desenvolvimento do patógeno pós-infecção.

Análises de correlação linear evidenciaram coeficientes de correlação altamente significativos $(\mathrm{P}<0,01)$ entre índice de severidade de doença (ISD) e características agronômicas como peso da panícula $\left(\mathrm{r}=-0,46^{* *}\right)$, número de grãos cheios $\left(\mathrm{r}=-0,35^{* *}\right)$, número de grãos chochos $\left(\mathrm{r}=0,45^{* *}\right)$, e porcentagem de esterilidade $\left(\mathrm{r}=0,50^{* *}\right)$. Não foram observadas correlações significativas entre qualquer um dos fungos patogênicos e a severidade de manchas nas sementes, evidenciando que todos os patógenos tiveram a mesma importância como agentes causais de manchas nas sementes, atuando como um complexo etiológico.
Avaliações das sementes produzidas em Capão Bonito, condições de terras altas irrigadas por aspersão:

Da mesma forma que observado nos experimentos instalados na outra localidade, a maioria das variáveis analisadas apresentou significância no Teste $\mathrm{F}$ para a interação ano X genótipo, motivo pelo qual foram considerados as análises e comparação de médias dentro de cada ano. Novamente as variáveis constituídas pela incidência dos fungos Bipolaris oryzae, Alternaria alternata e Nigrospora oryzae, e nesse caso também a variável constituída pelo índice de manchas nas sementes (ISD) não apresentaram interação significativa ano $\mathrm{X}$ genótipo, e foram considerados os resultados médios dos dois anos para a comparação de médias.

Os resultados dos testes de sanidade de sementes, apresentados na Tabela 3, evidenciam elevada incidência de $P$. grisea e Drechslera sp. no primeiro ano, com valores médios de 29,6 e $65,3 \%$, e acentuado decréscimo desses patógenos no segundo ano, com incidências médias de 4,9 e $24,6 \%$, respectivamente. Os demais fungos constatados apresentaram pouca variação entre os anos, com incidências médias anuais respectivas de 0,6 e $0,1 \%$ para $B$. oryzae, 0,1 e $0,6 \%$ para $M$. oryzae, 75,7 e 69,8 para P. sorghina; 1,6 e 0,0 para A. padwickii.

Houve variação entre os genótipos quanto ao comportamento como hospedeiros dos diversos fungos, e também entre os anos para um mesmo genótipo, podendo-se notar que genótipos que se destacaram no primeiro ano tiveram comportamento intermediário no segundo ano, e vice-versa. Destacou-se a linhagem IAC 1781 pelas menores incidências de $P$. grisea, P. sorghina, Drechslera spp., Curvularia spp. e Fusarium sp., seguida da cultivar Carisma pelas baixas incidências de P. sorghina e Drechslera spp.

O índice de severidade de doença - ISD - nas sementes (Tabela 4) novamente apresentou pequena amplitude de variação entre os genótipos, com valores médios nos 2 anos entre 1,29 a 1,87 (média de 
Tabela 3. Incidência dos principais fungos (\%) constatados em sementes de arroz, detectados através do teste de papel de filtro com congelamento. Capão Bonito, anos agrícolas 2002/2003 e 2003/2004.

\begin{tabular}{|c|c|c|c|c|c|c|c|c|c|c|c|c|c|c|c|c|c|c|}
\hline \multirow[b]{2}{*}{ Genótipos } & \multicolumn{18}{|c|}{ FUNGOS } \\
\hline & \multicolumn{3}{|c|}{ Pyricularia grisea } & \multicolumn{3}{|c|}{ Bipolaris oryzae } & \multicolumn{3}{|c|}{ Microdochium oryzae } & \multicolumn{3}{|c|}{ Phoma sorghina } & \multicolumn{3}{|c|}{ Drechslera spp. } & \multicolumn{3}{|c|}{ Alternaria padwicki } \\
\hline Bonança & 29,2 a-d & $10,5 \mathrm{a}$ & 19,9 & 0,1 & 0,1 & $0,1 \mathrm{a}$ & $0,0 \mathrm{a}$ & $2,0 \mathrm{a}$ & 1,0 & 78,7 a & 86,6 ab & 82,6 & $45,5 \mathrm{bc}$ & 25,6 a-e & 35,5 & 0,6 & 0,1 & $0,4 \mathrm{a}$ \\
\hline CNA 8983 & 37,0 a-d & $3,1 \mathrm{a}-\mathrm{c}$ & 20,1 & 1,3 & 0,3 & 0,8 a & 0,4 a & $1,4 \mathrm{a}$ & 0,9 & 79,0 a & $73,9 \mathrm{ab}$ & 76,5 & $64,8 \mathrm{ab}$ & 24,1 b-e & 44,4 & 2,5 & 0,1 & $1,3 \mathrm{a}$ \\
\hline IAC 1652 & 31,9 a-d & $3,3 \mathrm{a}-\mathrm{c}$ & 17,6 & 0,6 & 0,0 & $0,3 \mathrm{a}$ & $0,0 \mathrm{a}$ & $0,1 \mathrm{a}$ & 0,1 & 86,1 a & $65,3 \mathrm{a}-\mathrm{c}$ & 75,7 & $67,3 \mathrm{ab}$ & 22,6 b-e & 44,9 & 1,6 & 0,1 & $0,9 \mathrm{a}$ \\
\hline IAC 1711 & $16,1 \mathrm{~cd}$ & $1,8 \mathrm{a}-\mathrm{c}$ & 9,0 & 0,8 & 0,0 & $0,4 \mathrm{a}$ & $0,1 \mathrm{a}$ & $0,0 \mathrm{a}$ & 0,1 & $75,5 \mathrm{a}$ & 61,1 a-c & 68,3 & 77,4 a & $13,8 \mathrm{de}$ & 45,6 & 1,4 & 0,0 & $0,7 \mathrm{a}$ \\
\hline IAC 1738 & 36,6 a-d & $1,3 \mathrm{bc}$ & 19,0 & 0,6 & 0,0 & $0,3 \mathrm{a}$ & $0,1 \mathrm{a}$ & $0,3 \mathrm{a}$ & 0,2 & $81,1 \mathrm{a}$ & $79,1 \mathrm{ab}$ & 80,1 & $67,9 a b$ & $18,5 \mathrm{c}$-e & 43,2 & 1,0 & 0,0 & $0,5 \mathrm{a}$ \\
\hline IAC 1740 & 39,6 a-c & $11,6 \mathrm{a}$ & 25,6 & 1,6 & 0,1 & $0,9 \mathrm{a}$ & 0,4 a & 0,4 a & 0,4 & $77,9 \mathrm{a}$ & 83,9 ab & 80,9 & $67,8 \mathrm{ab}$ & $33,5 \mathrm{a}-\mathrm{c}$ & 50,6 & 1,9 & 0,1 & $1,0 \mathrm{a}$ \\
\hline IAC 1744 & 26,0 a-d & $7,9 \mathrm{a}-\mathrm{c}$ & 17,0 & 0,6 & 0,0 & $0,3 \mathrm{a}$ & 0,1 a & 0,6 a & 0,4 & 76,6 a & $86,1 \mathrm{ab}$ & 81,4 & $63,4 \mathrm{ab}$ & 31,0 a-d & 47,2 & 0,8 & 0,0 & $0,4 \mathrm{a}$ \\
\hline IAC 1774 & $16,1 \mathrm{~cd}$ & $4,3 \mathrm{a}-\mathrm{c}$ & 10,2 & 0,8 & 0,0 & 0,4 a & $0,3 \mathrm{a}$ & $0,1 \mathrm{a}$ & 0,2 & $62,4 \mathrm{a}$ & $62,9 \mathrm{a}-\mathrm{c}$ & 62,6 & 77,4 a & 19,0 c-e & 48,2 & 1,0 & 0,1 & $0,6 \mathrm{a}$ \\
\hline IAC 1776 & $15,5 \mathrm{~cd}$ & $3,0 \mathrm{a}-\mathrm{c}$ & 9,3 & 0,9 & 0,0 & 0,4 a & $0,1 \mathrm{a}$ & 0,1 a & 0,1 & $78,8 \mathrm{a}$ & $61,3 \mathrm{a}-\mathrm{c}$ & 70,0 & $72,8 \mathrm{a}$ & 22,1 b-e & 47,4 & 3,4 & 0,1 & $1,7 \mathrm{a}$ \\
\hline IAC 1779 & 29,1 a-d & $10,3 \mathrm{a}-\mathrm{c}$ & 19,7 & 0,3 & 0,1 & $0,2 \mathrm{a}$ & $0,0 \mathrm{a}$ & $1,4 \mathrm{a}$ & 0,7 & $75,4 \mathrm{a}$ & 87,1 ab & 81,2 & $71,5 \mathrm{ab}$ & $41,4 \mathrm{ab}$ & 56,5 & 1,5 & 0,1 & $0,8 \mathrm{a}$ \\
\hline$\underline{\text { Primavera }}$ & 19,6 b-d & $0,9 \mathrm{c}$ & 10,3 & 0,8 & 0,0 & $0,4 \mathrm{a}$ & $0,3 \mathrm{a}$ & $0,1 \mathrm{a}$ & 0,2 & $67,6 \mathrm{a}$ & $65,1 \mathrm{a}-\mathrm{c}$ & 66,4 & $69,5 \mathrm{ab}$ & 21,9 b-e & 45,7 & 1,4 & 0,0 & $0,7 \mathrm{a}$ \\
\hline Média & 29,6 & 4,9 & 17,2 & 0,6 & 0,1 & 0,3 & 0,1 & 0,6 & 0,4 & 75,7 & 69,8 & 72,8 & 65,3 & 24,6 & 44,9 & 1,6 & 0,0 & 0,8 \\
\hline CV (\%) & 25,5 & 34,7 & & & & 30,9 & 16,2 & 38,4 & & 10,5 & 10,6 & & 8,6 & 14,6 & & & & 45,5 \\
\hline
\end{tabular}

Médias seguidas da mesma letra, dentro de cada coluna, não diferem estatisticamente entre si pelo teste de Tukey a 5\%.

Tabela 4. Índice de severidade de manchas (ISD) e características agronômicas apresentadas por genótipos de arroz. Capão Bonito, anos agrícolas 2002/ 2003 e $2003 / 2004$.

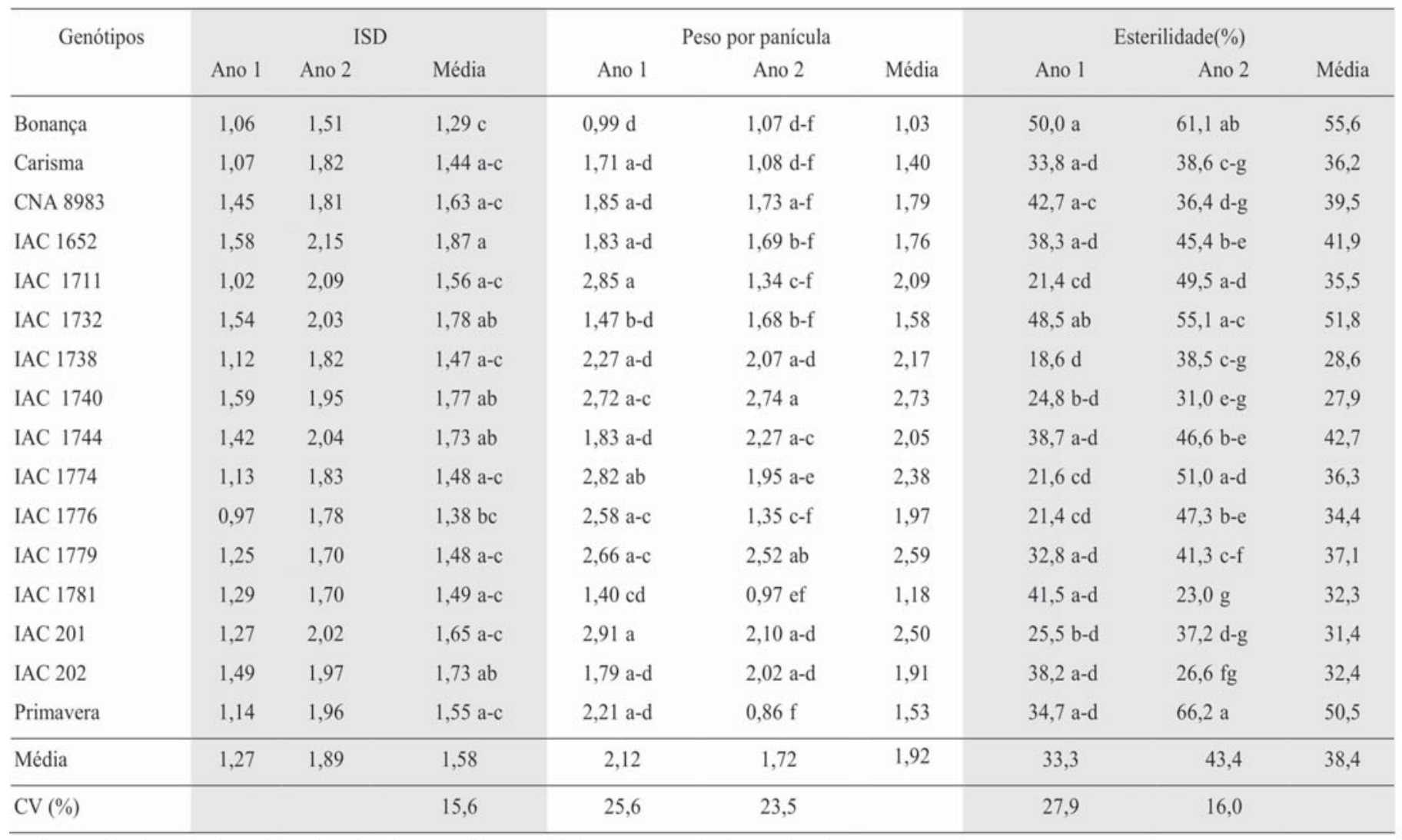

Médias seguidas da mesma letra, dentro de cada coluna, não diferem estatisticamente entre si pelo teste de Tukey a 5\%. 
1,58, escala de 0-4). Destacaram-se a cv. Bonança e a linhagem IAC 1776 pelos menores ISD. Pode-se observar que a severidade de manchas foi menor nesse sistema de cultivo em terras altas do que em relação ao anterior. Isso se deve provavelmente às baixíssimas incidências, quase nulas, de Bipolaris oryzae e Microdochium oryzae, patógenos de alta agressividade, agentes causais das doenças mancha parda e escaldadura, e dos mais importantes como causadores de manchas de grãos em arroz.

Os maiores pesos e grãos cheios por panícula foram apresentados por IAC 1740 , IAC 1774 , IAC 1779 , IAC 201, e as menores porcentagens de esterilidade por IAC 1740, IAC 201, IAC 1738, Carisma, IAC 1781 e IAC 202.

Com base nas avaliações dos dois anos nota-se que destacaram-se os genótipos IAC 1781, que apresentou baixa esterilidade e baixas incidências de cinco importantes patógenos, já relacionados anteriormente; IAC 1776, com baixo ISD e baixa incidência de $P$. grisea; IAC 1774, com alto peso e número de grãos por panícula e baixa incidência de $P$. grisea; Carisma, com baixa esterilidade e baixas incidências de P. sorghina e Drechslera spp.; e Bonança, com baixo ISD e baixa incidência de Curvularia sp.

Análises de correlação linear evidenciaram, à semelhança do ocorrido na outra localidade, coeficientes de correlação altamente significativos $(\mathrm{P} \leq 0,01)$ entre índice de severidade de doença (ISD) e características agronômicas como peso da panícula $\left(\mathrm{r}=-0,26^{* *}\right)$, número de grãos cheios $(\mathrm{r}=-0,32 * *)$, número de grãos chochos $(\mathrm{r}=$ $\left.0,25^{* *}\right)$, e porcentagem de esterilidade $(\mathrm{r}=0,33 * *)$. Novamente não foram observadas correlações significativas entre qualquer um dos fungos patogênicos e a severidade de manchas nas sementes.

\section{Os resultados do presente trabalho permitem concluir que:}

- A severidade de manchas nas sementes apresentou correlação negativa com peso da panícula, e positiva com porcentagem de esterilidade.

- Todos os patógenos tiveram a mesma importância como agentes causais de manchas, atuando como complexo etiológico.

- Em Taubaté, sob cultivo irrigado por inundação, destacaram-se as linhagens IAC 1817 e IAC 1818, apresentando os menores índices de severidade de manchas, além de baixas porcentagens de esterilidade e altos pesos por panícula.

- Em Capão Bonito, terras altas irrigadas por aspersão, destacaramse a linhagem IAC 1776 e a cv. Bonança, com os menores índices de severidade de manchas, e a linhagem 1781, com baixas incidências dos mais importantes patógenos, e também baixa esterilidade.

\section{REFERÊNCIAS BIBLIOGRÁFICAS}

1. Amaral, H.M. Testes de sanidade de sementes de arroz. In: soave, J.; wetzel, m.M.v.s.(Ed.). Patologia de sementes. Campinas, Fundação Cargill, 1987. p. 358-370.

2. Arias, S.M.S.; Filippi, M.C.; Bazoni, R.; Prabhu, A.S. Avaliação de linhagens de arroz de terras altas para resistência a mancha de grãos no Estado de Mato Grosso do Sul. Fitopatologia Brasileira, Brasília, v. 25, supl., p. 355, ago. 2000.

3. Cardoso, J.E. Doenças do arroz na microrregião Alto Purus Acre. CircularTecnica EMBRAPA-UEPAE, Rio Branco, n.1, 1980. $17 \mathrm{p}$.

4. Castano, J.; Klap, K.; Zaini, Z. Etiology of grain discoloration in upland rice in West Sumatra. International Rice Research Newsletter, Manila, v. 16, n. 1, p. 21, 1991.

5. Costa, J.L.S. Alternaria padwickii e Curvularia lunata: patogenicidade e transmissão por sementes de arroz irrigado. Fitopatologia Brasileira, Brasília, v. 16, n. 1, p. 15-18, 1991.
6. EMBRAPA. Centro de Pesquisa Agropecuária de Clima Temperado (Pelotas, RS). Arroz irrigado: recomendações técnicas da pesquisa para o Sul do Brasil. Documento EMBRAPA/CPACT., n.3, 1993. 87 p..

7. Groth, D.E.; Rush, M.C.; Hollier, C.A. Rice diseases and disorders in Louisiana. Baton Rouge: Louisiana Agricultural Experiment Station, 1991. 37p. (Bulletin, 828).

8. Kruppa, P. C.; Kohara, E. Y. ; Campos, G. W. Incidência de Alternaria padwickii, Rhynchosporium oryzae e Phoma sp. em sementes de arroz irrigado. Summa Phytopathologica, Jaboticabal, v. 24, n. 1, p. 55, 1998.

9. Malavolta, V.M.A.; Bedendo, I.P. Resistência de cultivares de arroz a manchas de grãos causadas pelos fungos Bipolaris oryzae, Microdochium oryzae e Phoma sorghina Summa Phytopathologica, Jaboticabal, v.25, n.4, p.313-318, 1999.

10. Malavolta, V.M.A.; Melo, E.A.; Vechi, V.A., Almeida, A.O. Comportamento de cultivares de arroz em relação à manchas de grãos. Summa Phytopathologica, Jaboticabal, v. 26, n. 1, p. 109, 2000 .

11. MAPA - Ministério da Agricultura, Pecuária e Abastecimento. CONAB. 2005. Disponível em <http://www.conab.gov.br/download/indicadores/0301-Oferta-e-demanda-brasileira.pdf $>$. Acesso em 17 nov. 2005.

12. MARA - Ministério da Agricultura e Reforma Agrária. Teste de sanidade de sementes. Regras para análise de sementes. Brasília, 1992. p. 204-212.

13. Ou, S.H. Rice diseases. 2 ed. Kew: Commonwealth Mycological Institute, 1985. 379p.

14. Prabhu, A.S.; Bedendo, I.P. Glume blight of rice in Brazil: etiology, varietal reaction and loss estimates. Tropical Pest Management, London, v. 34, n.1, p.85-88, 1988.

15. Ribeiro, A.S. Controle integrado das doenças do arroz irrigado. Circular Técnica EMBRAPA/CPATB, n.3, 1989. 29p.

16. Ribeiro, A.S.; Tanaka, M.A.S. Doenças do arroz e medidas de controle. Informe Agropecuário, Belo Horizonte, v. 10, n. 114, p. 24-32, 1984 .

17. Santos, G.R.; Collicchio, E.; Guimarães, H.M.A. Avaliação da resistência de genótipos de arroz a mancha-de-grãos no município de Formoso do Araguaia, Estado do Tocantins. Fitopatologia Brasileira, Brasília, v. 21, sup1., p. 382, ago. 1996.

18. Santos, G.R.; Costa, W.M.; Costa, H. Incidência de mancha parda do arroz no projeto Formoso, Estado do Tocantins. Fitopatologia Brasileira, Brasília, v.19, supl., p. 299, ago. 1994.

19. Santos, G.R.; Santos, J. Incidência de doenças em arroz irrigado no Estado de Tocantins. Fitopatologia Brasileira, Brasília, v. 20, supl., p. 345, ago. 1995.

20. Silva, V.A.; Cornélio, V.M.O.; Soares, A.A. Ocorrência de fungos em genótipos de arroz e sua influência sobre o rendimento de inteiros e peso de 100 grãos. Fitopatologia Brasileira, Brasília, v. 25, supl., p. 453, ago. 2000.

21. Singh, B.M.; Saharan, G.S.; Shyam, K.R.; Sood, A.K. Factors affecting severity of glume blight in different cultivars of rice in Himachal Pradesh. Indian Phytopathology, Nova Delhi, v. 31, p. 419-423, 1978.

22. Soave, J.; Pizzinatto, M.A.; Usberti Júnior, J.A.; Camargo, O.B.A.; Villela, O.V. Selection of rice cultivars resistant to some pathogens using seed health testing. Pesquisa Agropecuária Brasileira, Brasília, v. 19, n.4, p. 449-453, 1984.

23. Soave, J.; Prabhu, A.S. ; Ricci, M.T.T.; Barros, L.G.; Souza, N. R. G.; Curvo, R. C. V.; Ferreira, R. P. ; Sobral, C.A.M. Etiologia de manchas de sementes de cultivares de arroz de sequeiro no Centro - Oeste brasileiro. Summa Phytopathologica, Jaboticabal, v. 23, p. 122-127, 1997.

24. Souza, N.R.G.; Curvo, R.C.; Prabhu, A.S.; Barros, L.G. Ocorrência e severidade de doenças do arroz de sequeiro no Estado do Mato Grosso. In: Reunião Nacional de Pesquisa de Arroz, 3., Goiânia, 1987. Anais. Goiânia: EMBRAPA, CNPAF, 1987. p. 483-509.

25. Souza, N.S.; Abreu, J.G.; Prabhu, A.S. Resistência de cultivares e linhagens de arroz a mancha de grãos. Fitopatologia Brasileira, Brasília, v. 20, supl., p. 346, ago. 1995.

26. Souza, N.S.; Barros, L.G.; Prabhu, A.S. Incidência de mancha 
de grãos do arroz em relação à época de plantio e aplicação de fungicidas. Boletim Pesquisa EMPAER, Várzea Grande, n.4, 1994. $19 \mathrm{p}$.

27. Souza, N.S.; Zambolim, L. Resistência varietal do arroz (Oryza sativa) à queima das glumelas (Phoma sorghina). Fitopatologia Brasileira, Brasília, v. 12, n. 1, p. 50-52, 1987.

28. Urben, A.F.; Wetzel, M.M.V.S. Ocorrência, sobrevivência e controle de Phyllosticta oryzae em sementes de arroz produzi- do nas condições dos cerrados. Fitopatologia Brasileira, Brasília, v. 5, n. 3, p. 462, 1980.

29. Zonta, E.P.; Machado, A.A.; Silveira Júnior, P. Sistema de análise estatística para microcomputadores. Manual de utilização. 2.ed., Pelotas: UFP, 1987. 177p.

30. Zulkifli, E. ; Klap, J. ; Castano, J. Effect of grain discoloration in upland rice on some yield components. International Rice Research Newsletter, Manila, v. 16, n. 4, p. 20, 1991 